\title{
A zero-cumulant random variable and its applications
}

\author{
Nicolas Petrochilos ${ }^{\mathrm{a}, *}$, Pierre Comon ${ }^{\mathrm{b}}$ \\ ${ }^{a}$ DISP, Università di Roma II Tor Vergata, Via delPolitecnico, 1, I-00173 Rome, Italy \\ ${ }^{\mathrm{b}}$ I3S, CNRS, University of Nice, 2000 route des Lucioles, BP 121, 06903 Sophia-Antipolis, France
}

Received 14 December 2004; received in revised form 8 October 2005; accepted 13 February 2006

Available online 10 March 2006

\begin{abstract}
High Order Statistics (HOS) are widely used in many algorithms ranging from blind identification to signal separation. A well-known identifiability result is that at most one Gaussian source should be present in static mixtures [J. Eriksson, V. Koivunen, Identifiability, separability and uniqueness of linear ICA models, IEEE Signal Process. Lett. (2004) 601-604]. The reason for this is that these algorithms utilize cumulants of order higher than two, and that they are all null for circular Gaussian random variables [M. Kendall, A. Stuart, The Advanced Theory of Statistics, Distribution Theory, vol. 1, C. Griffin, 1977]. Simple examples of non-Gaussian complex random variables having zero cumulants of order three to seven are given, which can be encountered in the real world.
\end{abstract}

(C) 2006 Elsevier B.V. All rights reserved.

Keywords: High-order statistics; Zero constant modulus; Kurtosis; Pseudo-Gaussian distributions

\section{Introduction}

Many practical algorithms use High Order Statistics (HOS), possibly non-circular, to perform blind signal separation, blind or data-aided identification, or linear and non-linear filtering [1,3-6]. Due to the use of HOS, at most one Gaussian source can be accepted in static mixtures (identifiability result from [1]). Actually, most of these numerical algorithms do not use cumulants of order higher than fourth. Therefore a distribution, which

\footnotetext{
${ }^{2}$ Part of this work was initiated at CAS, Delft University of Technology, The Netherlands.

*Corresponding author. Present address: CReSTIC/GSMA, University of Reims, Moulins de la Housse, BP 1039, 51687 Reims Cedex 2, France. Tel.: + 33326918219 ; fax: +33326913106 .

E-mail addresses: nicolas.petrochilos@univ-reims.fr (N. Petrochilos), pcomon@i3s.unice.fr (P. Comon).
}

third and fourth order cumulants are zeros, behaves as Gaussian from the point of view of the algorithm.

It is known that the only probability distribution having a finite number of non-zero cumulants is Gaussian. Reversely, having only a few cumulants fixed permits to generate many families of distribution. However, there are very few examples given in the literature of non-Gaussian distributions having null fourth or sixth order cumulants [2]. Users of HOS should not think that it is sufficient to check the first cumulants in order to make sure that a distribution is Gaussian or not.

We present a simple non-Gaussian complex distribution, called Zero Constant Modulus (ZCM), whose cumulants of order three-five are all null [7]. Thus, this random variable exhibits an apparent Gaussianity up to order five. Although this may appear as a curiosity to some readers, these variables are encountered in various practical 
problems, as pointed out subsequently. In addition, we pushed further the academic exercise to cancel all cumulants up to sixth order.

Notation. Denote moments of zero-mean complex random variables as

$\mu_{p}^{q}=E\left\{s^{p} s^{* q}\right\}$.

For instance, moment $\mu_{1}^{1}$ corresponds to the variance. Also denote $\kappa_{p}^{q}=\operatorname{Cum}\left\{s, \ldots, s, s^{*}, \ldots, s^{*}\right\}$ their cumulants of order $p+q$, where $q$ denotes the number of complex conjugated terms. Recall that fourth order cumulants are related to moments via simple relations [2]

$\kappa_{2}^{2}=\mu_{2}^{2}-2\left(\mu_{1}^{1}\right)^{2}-\mu_{2}^{0} \mu_{0}^{2}$,

$\kappa_{3}^{3}=\mu_{3}^{3}-9 \mu_{2}^{2} \mu_{1}^{1}+12\left(\mu_{1}^{1}\right)^{3}$,

where $\kappa_{2}^{2}$ is referred to as the kurtosis. ${ }^{1}$

\section{The ZCM distribution}

Let $s[n]$ be a random variable, whose values are either 0 , or on the unit circle, with equal probabilities:

$P(s=0)=\frac{1}{2}=P(|s|=1)$

and with an uniform distribution over the unit circle; hence the name of ZCM. The expected mean is then

$\mu_{1}^{0}=\int s P(s) \mathrm{d} s=0 \times \frac{1}{2}+\left(\oint_{\mathscr{C}} s \frac{1}{2 \pi} \mathrm{d} s\right) \frac{1}{2}=0$,

where $\mathscr{C}$ is the unit circle. As the distribution is invariant by any rotation in the complex plane, this distribution is circular [8]. Similarly, because of circularity, we have

$\mu_{2}^{0}=0, \quad \mu_{3}^{0}=\mu_{2}^{1}=0, \quad \mu_{4}^{0}=\mu_{3}^{1}=0$.

Note that all non-circular moments (i.e. moments $\mu_{k}^{\ell}$ with $k \neq \ell$ ) of higher order are also null. On the other hand, the circular ${ }^{2}$ moments are equal to

$\mu_{k}^{k}=\int s^{k} s^{* k} P(s) \mathrm{d} s=0 \times \frac{1}{2}+1 \times \frac{1}{2}=\frac{1}{2}$

for any $k \in \mathbb{N}$. In particular, the variance $\mu_{1}^{1}$ and the circular fourth-order moment $\mu_{2}^{2}$ are non-zero.

It turns out that cumulants of order three-five of $s$ are all equal to zero, including the kurtosis (2),

\footnotetext{
${ }^{1}$ The standardized cumulant $\kappa_{2}^{2} /\left(\kappa_{1}^{1}\right)^{2}$ is also sometimes called kurtosis.

${ }^{2}$ Moments where exactly half of the variables are complex conjugated are sometimes referred to as "circular" moments.
}

which takes the simpler expression: $\kappa_{2}^{2}=\mu_{2}^{2}-$ $2\left(\mu_{1}^{1}\right)^{2}=0$. Thus, without resorting to statistics of order higher than 5, a ZCM variable is perceived as a Gaussian variable.

Note that by multi-linearity of cumulants, any linear combination of ZCM variables, $\mathbf{y}=\sum_{i} a_{i} s_{i}$, will still have all its cumulants of order 3,4 and 5 all equal to zero. It is then not reasonable to trust methods based only on these cumulants.

\section{A radar application}

More than an interesting exercise, this distribution can be met in real-life situations, and for example secondary surveillance radars (SSR). Secondary surveillance radar (SSR) is essential for air traffic control (ATC). Unlike primary radar, its principle is not based on the detection of the reflection of an electromagnetic wave, but rather on a communication between the radar and the aircraft $[9,10]$. An onboard device, the transponder, receives a request from the radar, and transmits the reply. The protocol of this radar is evolving from the "Mode A/C" [11] toward the "Mode S". The new communication protocol consists of a preamble followed by a pulse train containing either 56 or 112 binary bits. The data bits are encoded in a "Manchester Encoding" scheme, which means that a bit $b_{n}=0$ is coded as $\mathbf{b}_{n}=[0,1]$, and a bit $b_{n}=1$ as $\mathbf{b}_{n}=[1,0]$. The signal is sampled at twice the data-rate: $f_{\mathrm{S}}=T^{-1}=2 \mathrm{MHz}$, and the length of a reply is $N=\{128,240\}$. Due to a tolerance on the carrier frequency, a residual frequency $f$ remains after down-conversion to baseband, and several phase rotations appear, as shown in Fig. 1. In this figure, the effect of the frequency tolerance is visible, and the distribution of the received signal fits a ZCM distribution. As a consequence, the received SSR signal has a distribution that can be assimilated to ZCM.

\subsection{Statistical behavior of SSR replies}

Given the specifications, the probability to get a zero is equal to 0.516 , and the probability to obtain a value on the unit circle is 0.484 . The probability distribution on the unit circle depends of the product $N f T$, as this product represents the number of loops the signal will do on the unit circle. The larger this value is, the more regular on the unit circle the probability distribution. A critical value for the frequency is given by the condition $N f T=1$ 


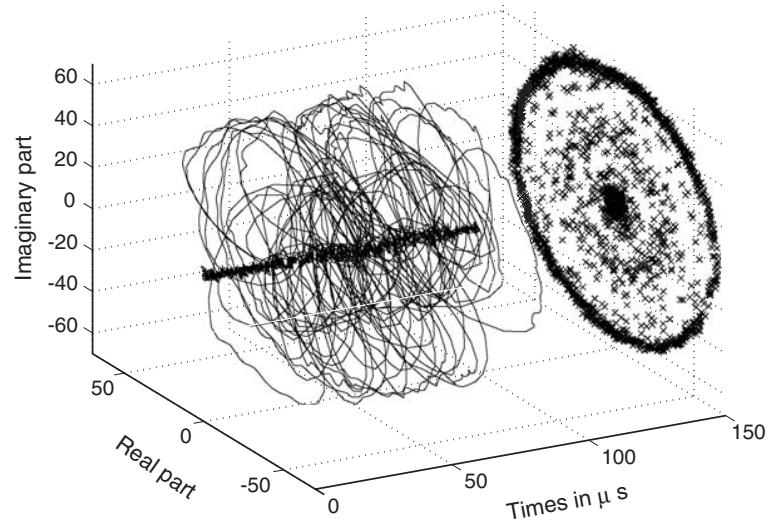

Fig. 1. A real measured "Mode S" reply is presented here with the associated probability distribution.

and corresponds to the case where the remaining frequency induces exactly one full rotation on the unit circle. Below this value, the reply cannot be assimilated as a ZCM distribution as only a portion of the unit circle is used; for instance a short mode $\mathrm{S}$ (56 bits) is behaving as a ZCM distribution for a frequency equal to $17 \mathrm{kHz}$, over a carrier frequency of $1 \mathrm{GHz}$ with a $3 \mathrm{MHz}$ tolerance.

For sake of simplicity, we consider a simplified model: let us reduce the replies to the informative part (without the preamble), and with an unitary gain $(g=1)$. We perform the calculation of the various sample moments, which leads to

$$
\begin{aligned}
& E\left\{\hat{\mu}_{1}\right\}=E\left\{\hat{\mu}_{21}\right\}=\frac{1}{2 N} \frac{\phi^{N}-1}{\phi-1}, \\
& E\left\{\hat{\mu}_{20}\right\}=\frac{1}{2 N} \frac{\phi^{2 N}-1}{\phi^{2}-1} \\
& E\left\{\hat{\mu}_{11}\right\}=\frac{1}{2}, \quad E\left\{\hat{\mu}_{22}\right\}=\frac{1}{2} .
\end{aligned}
$$

As a consequence, the estimation of kurtosis $\hat{\kappa}_{22}$, defined as a function of sample moments by

$$
\begin{aligned}
\hat{\kappa}_{22}= & \hat{\mu}_{22}-2\left(\hat{\mu}_{1} \hat{\mu}_{12}+\hat{\mu}_{1}^{*} \hat{\mu}_{12}^{*}\right)-\left|\hat{\mu}_{20}\right|^{2}-2 \hat{\mu}_{11}^{2} \\
& +8\left|\hat{\mu}_{1}\right|^{2} \hat{\mu}_{11}+2\left(\hat{\mu}_{1}^{2} \hat{\mu}_{02}+\hat{\mu}_{1}^{* 2} \hat{\mu}_{02}^{*}\right)-6\left|\hat{\mu}_{1}\right|^{4}
\end{aligned}
$$

has an expected mean

$$
E\left\{\hat{\kappa}_{22}\right\}=-\left[2\left|E\left\{\hat{\mu}_{1}\right\}\right|^{4}+\left|2 E\left\{\hat{\mu}_{1}\right\}^{2}-E\left\{\hat{\mu}_{20}\right\}\right|^{2}\right] .
$$

Fig. 2 shows the theoretical value of $\kappa_{22}$ as a function of the residual carrier, its estimated average over 1000 independent runs, and its standard deviation. For frequencies above $f=17 \mathrm{kHz}$, the expected average is negligible compared to its standard deviation, which confirms the kurtosis vanishing.

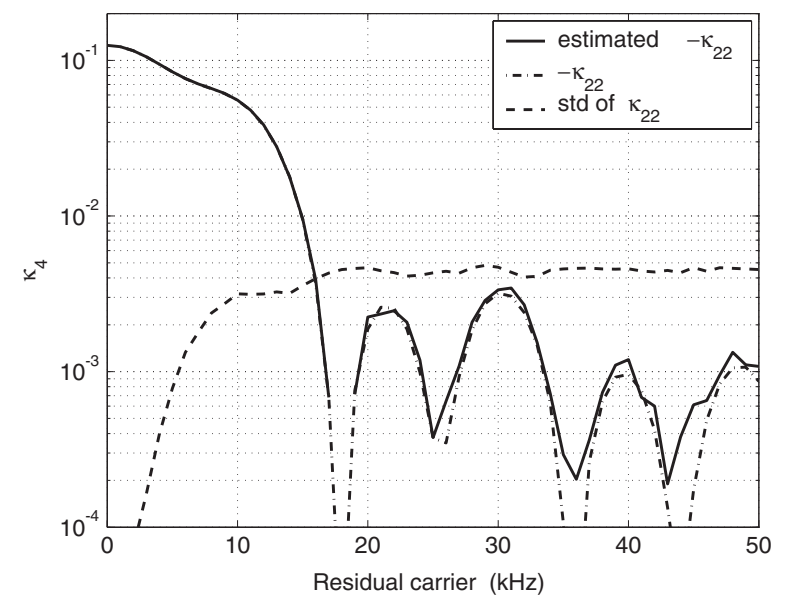

Fig. 2. Mean and standard deviation of $\kappa_{22}$ as a function of the residual carrier. The simulation are done with packets of 112 samples, over 1000 independent runs.

Fig. 3 shows the sum of the absolute values of the cumulants used by the algorithm CoM [3] as a function of the residual carriers of two sources. The simulation are performed with packets of 112 samples, over 1000 independent runs.

We note that several zones are black, meaning that the used cumulants are quite below the estimation noise. Moreover, only a small part of the range of possible frequencies is shown; the brightest. Given that the carrier drifts are a priori unknown, even if the SSR replies are not all behaving as a ZCM sources as described above, it is reasonable to avoid the kind of algorithms as CoM, or JADE $[12,13]$ to separate such sources.

The algorithm CoM has been applied successfully to the previous protocol, the "mode A/C" (see [11]). This is not a contradiction to our result as (i) this protocol had only 42 samples, and (ii) the coding of the data was different, leading to a probability to get a 0 quite larger than 0.5. Consequently, cumulants vanish for much higher residual frequencies, and the probability to obtain an almost full overlap is very weak; HOS algorithms have thus better working conditions, and the results from [11] are still valid.

Another case of application can be found in other On-Off Keyings (OOK) in the presence of frequency offset.

\section{Cumulants of 6th order}

It is now natural to wonder whether this result could be extended to higher orders. It turns out that 


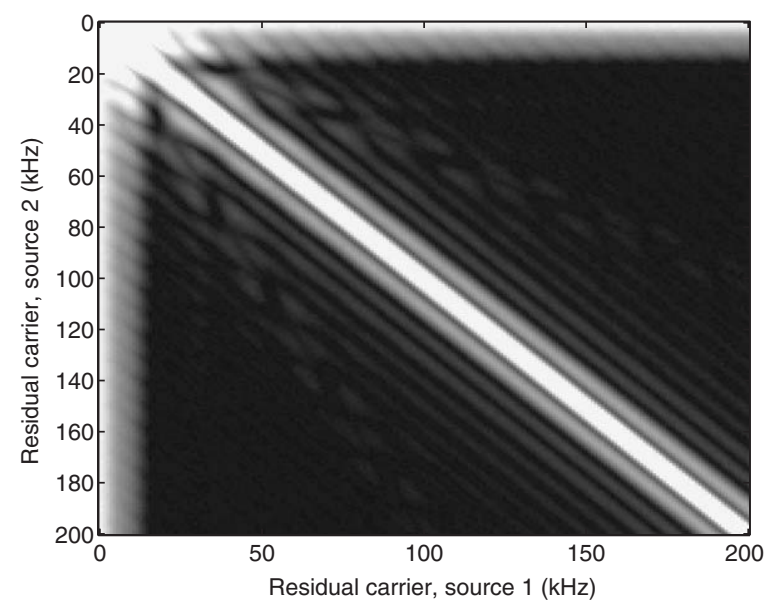

Fig. 3. Sum of the absolute values of the cumulants used by the method CoM [3] as a function of the source residual carriers.

the answer is yes, and we give here an explicit answer for order six. First, it appears that all sixth order cumulants of ZCM are null except the circular cumulant below:

$\kappa_{3}^{3}=\frac{7}{8}$.

Now, consider a random variable whose values are taken equal to 0 with probability $\alpha$, or taken on a circle of radius $r$ with probability $\beta$, or taken on a circle of radius $d r$ with probability $\gamma, d>1$. The circular moments are for any $k \in \mathbb{N}$ :

$\mu_{k}^{k}=r^{2 k}\left(\beta+\gamma d^{2 k}\right)$.

Because the distribution is circular, i.e. invariant by rotation in the complex plane, all cumulants up to order seven are equal to zero, except maybe circular cumulants $\kappa_{1}^{1}, \kappa_{2}^{2}$, and $\kappa_{3}^{3}$.

Parameter $r$ appears just as a scale parameter, and has no influence on standardized cumulants, which can be expressed as a function of $d$ only:

$\kappa_{2}^{2}=f(\beta, \gamma, d)$,

$\kappa_{3}^{3}=g(\beta, \gamma, d)$,

where $g($.$) and f($.$) are multivariate polynomials of$ degrees 4 and 6 , respectively. Forcing $\kappa_{2}^{2}=0$ and $\kappa_{3}^{3}=0$ yields a system of two polynomial equations in three variables; there are thus generally infinitely many solutions described by the union of several one-parameter curves. This set can be restricted, due to the constraints of positivity of the probabilities, and of their sum to 1 . Several solutions are plotted in Fig. 4. This defines a whole family of simple nonGaussian distributions having zero cumulants up to order seven.

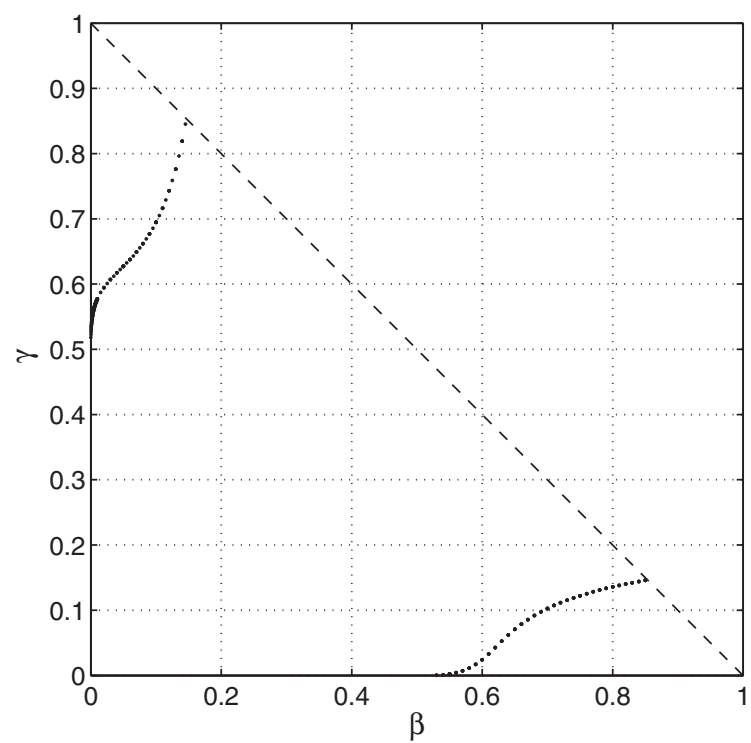

Fig. 4. Some solution points in the $(\beta, \gamma)$ plane.

\section{Conclusions and perspectives}

Even if non-Gaussian distributions having zero cumulants of order higher than two are known to exist, they were not really considered as likely to occur, and no simple example was provided in the literature, even in textbooks. We have first presented a non-Gaussian distribution behaving as Gaussian up to order five, namely the ZCM distribution. This distribution is found in real-life situations, such as in Secondary Surveillance Radar (SSR). Many other "pseudo-Gaussian" distributions can be derived arbitrarily. As an example among other, a simple family of complex distributions has been proposed, having null cumulants up to order seven.

\section{References}

[1] J. Eriksson, V. Koivunen, Identifiability, separability and uniqueness of linear ICA models, IEEE Signal Process. Lett. (2004) 601-604.

[2] M. Kendall, A. Stuart, The Advanced Theory of Statistics, Distribution Theory, vol. 1, C. Griffin, 1977.

[3] P. Comon, Independent component analysis, a new concept?, Signal Processing, Elsevier 36 (3) (1994) 287-314 (Special issue on Higher-Order Statistics).

[4] J.-C. Pesquet, E. Moreau, Cumulant based independence measures for linear mixtures, IEEE Trans. Inform. Theory (2001) 1947-1956. 
[5] C.L. Nikias, A.P. Petropulu, Higher-order Spectra Analysis: A Nonlinear Signal Processing Framework, PTR PrenticeHall, Englewood Cliffs, NJ, 1993.

[6] E. Moreau, A generalization of joint-diagonalization criteria for source separation, IEEE Trans. Signal Process. 49 (3) (2001) 530-541.

[7] N. Petrochilos, P. Comon, Separation de signaux ZCM: application en radar SSR, in: Proceedings of GRETSI 2003, Paris, France, 2003.

[8] P.O. Amblard, M. Gaeta, J.L. Lacoume, Statistics for complex variables and signals, part I: variables, Signal Process. 53 (1996) 1-13.

[9] M. Stevens, Secondary Surveillance Radar, Artech House, Norwood, MA, 1988.
[10] N. Petrochilos, Algorithms for Separation of Secondary Surveillance Radar Replies, Doctorate, Universities de NiceSophia-Antipolis and TU Delft, Nice, France, ISBN 90-4072371-0, www.i3s.unice/ petro (July 2002).

[11] E. Chaumette, P. Comon, D. Muller, An ICA-based technique for radiating sources estimation; application to airport surveillance, IEE Proceedings - Part F 140 (6) (1993) 395-401 (Special issue on Applications of High-Order Statistics).

[12] J.-F. Cardoso, A. Souloumiac, Blind beamforming for nonGaussian signals, IEE Proc.-F 140 (6) (1993) 362-370.

[13] J.-F. Cardoso, A. Souloumiac, Jacobi angles for simultaneous diagonalization, SIAM J. Matrix Anal. Appl. 17 (1) (1996) 161-164. 